\title{
Multi-Step Analysis of Diol-LC-ESI-HRMS Data Reveals Proanthocyanidin Composition of Complex Plant Extracts (PAComics)
}

\author{
Nico Symma, ${ }^{1}$ Jandirk Sendker, ${ }^{1}$ Frank Petereit, ${ }^{1}$ and Andreas Hensel*,1 \\ ${ }^{1}$ Institute of Pharmaceutical Biology and Phytochemistry, University of Münster, \\ Corrensstraße 48, D-48149 Münster, Germany
}




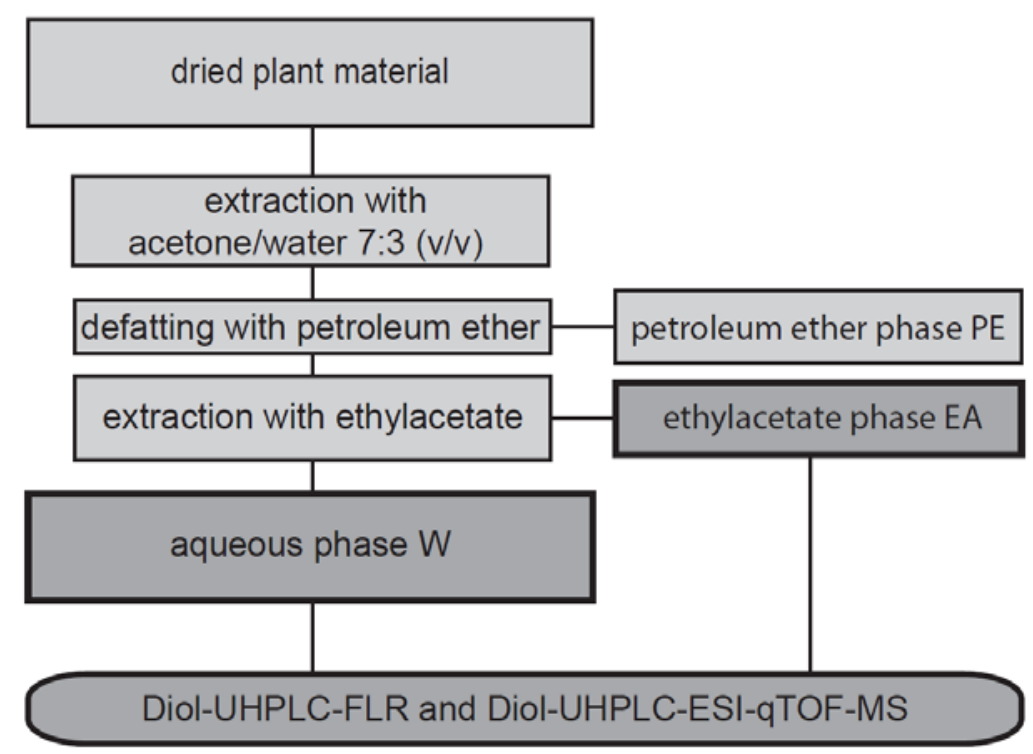

Figure S-1. Preparation of proanthocyanidin (PAC)-enriched plant extracts. PAC with low degree of polymerisation (DP) can be found in the ethylacetate phase EA. Oligomeric PAC and polymers remain in the aqueous phase $\mathrm{W}$. 
(I) $M / u=m / z \cdot \mid$ charge state $\mid-($ charge state $\cdot 1,0073)$

(II) Kendrick factor $\left(F_{K}\right)=\frac{\text { nominal mass [building block] }}{\text { accurate mass [building block] }}=\frac{\text { nominal mass [(epi)catechin] }}{\text { accurate mass [(epi)catechin] }}=\frac{288}{288,0634}=0.99978$

(III) Kendrick mass (KM) $=M / u \cdot F_{K}$

(IV) Kendrick mass defect (KMD) $=$ nominal $\mathrm{KM}-$ accurate KM

Figure S-2. Calculation of Kendrick mass defect (KMD) from the nominal mass and the accurate mass as determined by HRMS for oligomeric proanthocyanidins, consisting of (epi)catechin building blocks ( $M / u$ : molecular mass $[\mathrm{u}])$. 


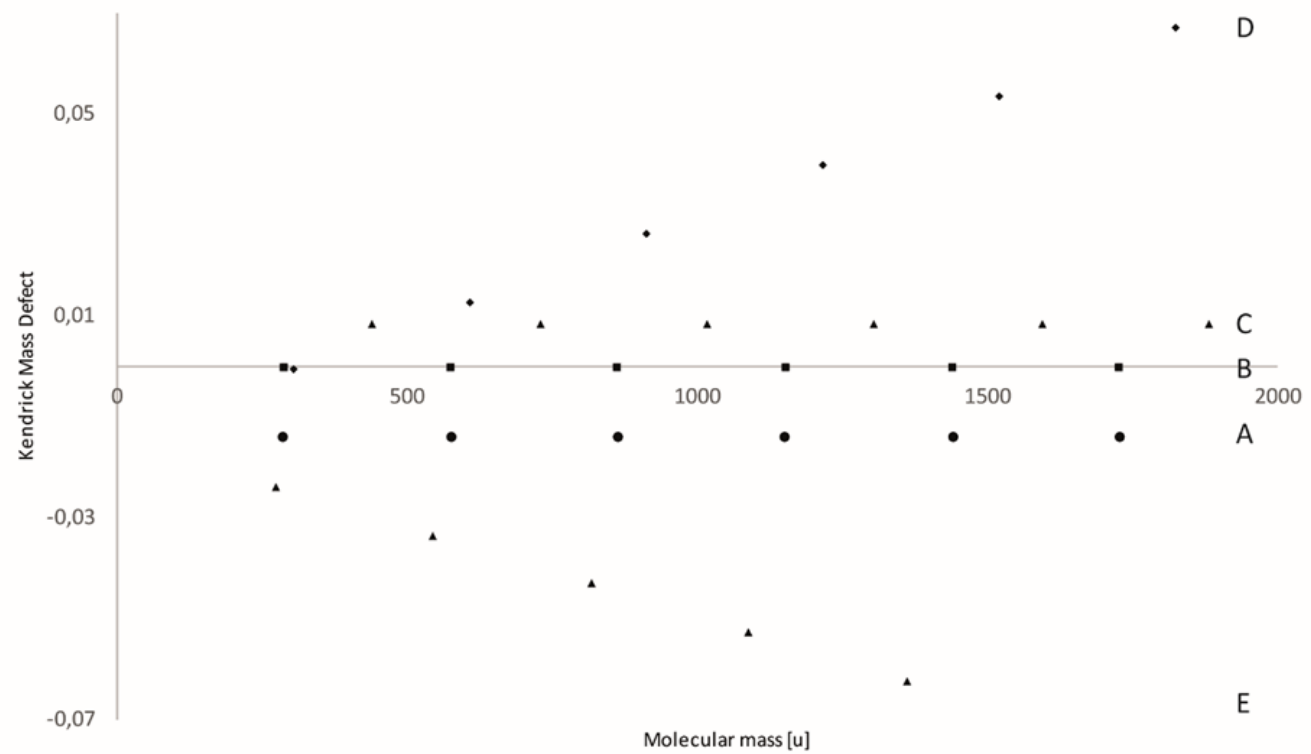

Figure S-3: Exemplary KMD plots of theoretical PAC oligomers DP 1 to 5; (A) PAC oligomers consisting exclusively of B-type (epi)catechin; (B) PAC oligomers consisting of B-type (epi)catechin interflavan linkage with one A-type interflavan linkage; (C) PAC oligomers consisting of B-type (epi)catechin with one additional gallic acid esterification; (D) PAC oligomers consisting of B-type epicatechin with 1 to 5 additional gallic acid ester residues; (E) PAC DP5, consisting exclusively of B-type (epi)gallocatechin. 
Table S-1. Dry extract yields [\% w/w, related to the dried plant material], obtained from different plant materials after extraction with acetone:water $(7: 3 \mathrm{~V} / \mathrm{V})$, removal of solvents, lyophlization and preparation of ethylacetate-soluble (EA) dry extract and water-soluble extract (W).

\begin{tabular}{|l|c|c|}
\hline \multicolumn{1}{|c|}{ Herbal material } & $\begin{array}{c}\text { Yield [\% w/w] } \\
\text { Fraction EA }\end{array}$ & $\begin{array}{c}\text { Yield [\% w/w] } \\
\text { Fraction W }\end{array}$ \\
\hline Hawthorn leaf and flower & 3.2 & 14.9 \\
\hline Lime flower & 3.8 & 14.8 \\
\hline Sorrel herb & 2.0 & 6,5 \\
\hline Japanese Wisteria fruits without seeds & 5.0 & 6.4 \\
\hline
\end{tabular}


Table S-2. Calculated mass defects and Kendrick mass defects for B-type proanthocyanidin polymers consisting of (epi)catechin. Whereas the mass defect is decreasing with increasing DP, the Kendrick mass defect remains the same.

\begin{tabular}{|l|l|l|l|l|l|l|l|}
\hline DP & $\begin{array}{c}\text { Molecular } \\
\text { formula }\end{array}$ & $\begin{array}{c}\text { Calculated } \\
\text { molecular } \\
\text { mass [u] }\end{array}$ & $\begin{array}{c}\text { Round } \\
\text { molecular } \\
\text { mass [u] }\end{array}$ & $\begin{array}{c}\text { Mass } \\
\text { defect }\end{array}$ & $\begin{array}{c}\text { Kendrick } \\
\text { mass [u] }\end{array}$ & $\begin{array}{c}\text { Round } \\
\text { Kendrick } \\
\text { mass [u] }\end{array}$ & $\begin{array}{c}\text { Kendrick } \\
\text { mass } \\
\text { defect }\end{array}$ \\
\hline $\mathbf{1}$ & $\mathrm{C}_{15} \mathrm{H}_{14} \mathrm{O}_{6}$ & 290,078 & 290 & $-0,078$ & 290,0141566 & 290 & $-0,0142$ \\
\hline $\mathbf{2}$ & $\mathrm{C}_{30} \mathrm{H}_{26} \mathrm{O}_{12}$ & 578,1414 & 578 & $-0,1414$ & 578,0141566 & 578 & $-0,0142$ \\
\hline $\mathbf{3}$ & $\mathrm{C}_{45} \mathrm{H}_{38} \mathrm{O}_{18}$ & 866,2048 & 866 & $-0,2048$ & 866,0141566 & 866 & $-0,0142$ \\
\hline $\mathbf{4}$ & $\mathrm{C}_{60} \mathrm{H}_{45} \mathrm{O}_{24}$ & 1154,2682 & 1154 & $-0,2682$ & 1154,014157 & 1154 & $-0,0142$ \\
\hline $\mathbf{5}$ & $\mathrm{C}_{75} \mathrm{H}_{62} \mathrm{O}_{30}$ & 1442,3316 & 1442 & $-0,3316$ & 1442,014157 & 1442 & $-0,0142$ \\
\hline $\mathbf{6}$ & $\mathrm{C}_{90} \mathrm{H}_{74} \mathrm{O}_{36}$ & 1730,395 & 1730 & $-0,395$ & 1730,014157 & 1730 & $-0,0142$ \\
\hline
\end{tabular}


Table S-3. LCMS/MS data from DP3 PAC series in the ethylacetate EA fractions from different plant extracts; GF: gallate fission, QM: quinone methide (= interflavan cleavage), HRF: heterocyclic ring fission; RDA: Retro-Diels-Alder; *: $\mathrm{m} \Sigma$ is not applicable, because isotopic pattern of A-type PACs are interfering with the signals of B-type PACs

\begin{tabular}{|c|c|c|c|c|c|c|c|c|c|}
\hline Plant material & PAC class & Ion formula & $t_{\mathrm{R}} / \min$ & $m / z$ & $\begin{array}{l}\text { error / } \\
\text { mDa }\end{array}$ & $\mathrm{m} \Sigma$ & $\begin{array}{l}\text { Intensity } \\
\text { of the } \\
\text { isotope } \\
\text { signal } \\
\text { [cnts] }\end{array}$ & diagnostic fragments (neutral loss) $[30 \mathrm{eV}]$ & $\begin{array}{l}\text { Proposed PAC } \\
\text { chain }\end{array}$ \\
\hline \multirow{3}{*}{ Tilia EA } & B-type & {$\left[\mathrm{C}_{45} \mathrm{H}_{39} \mathrm{O}_{18}\right]^{+}$} & 9.441 & 867.2085 & -4.6 & 7.2 & 37814 & $\begin{array}{l}\left.741 \text { (126; HRF), } 715 \text { (152; RDA), } 697 \text { (152, 18; RDA, } \mathrm{H}_{2} \mathrm{O}\right), 579 \\
(288 ; \mathrm{QM} \uparrow), 577 \text { (290; QM } \downarrow), 451(126,290 ; \mathrm{HRF}, \mathrm{QM} \downarrow), 427 \\
(288,152 ; \mathrm{QM} \uparrow, \mathrm{RDA}), 425(290,152 ; \mathrm{QM} \downarrow, \mathrm{RDA}), 409(288, \\
\left.152,18 ; \mathrm{QM} \uparrow, \mathrm{RDA}, \mathrm{H}_{2} \mathrm{O}\right), 407(290,152,18 ; \mathrm{QM} \downarrow, \mathrm{RDA}, \\
\left.\mathrm{H}_{2} \mathrm{O}\right), 291(576 ; \mathrm{QM} \uparrow), 289(578 ; \mathrm{QM} \downarrow)\end{array}$ & $\begin{array}{l}\text { (epi)cat-(epi)cat- } \\
\text { (epi)cat }\end{array}$ \\
\hline & A-type & {$\left[\mathrm{C}_{45} \mathrm{H}_{37} \mathrm{O}_{18}\right]^{+}$} & 8.554 & 865.1974 & -3.7 & $-*$ & 6504 & $\begin{array}{l}\left.\left.713 \text { (152; RDA), } 695 \text { (152, 18; RDA, } \mathrm{H}_{2} \mathrm{O}\right), 577 \text { (288; QM } \uparrow\right), \\
\left.407 \text { (288, 152, 18; QM } \uparrow, \mathrm{RDA}, \mathrm{H}_{2} \mathrm{O}\right), 289(576 ; \mathrm{QM} \downarrow), 287 \text { (288, } \\
290 ; \mathrm{QM} \uparrow, \text { A-type-QM } \downarrow)\end{array}$ & $\begin{array}{l}\text { (epi)cat-(epi)cat- } \\
\text { A-(epi)cat }\end{array}$ \\
\hline & $\begin{array}{l}\text { B-type with } \\
\text { one } \\
\text { (epi)afzelechin }\end{array}$ & {$\left[\mathrm{C}_{45} \mathrm{H}_{39} \mathrm{O}_{17}\right]^{+}$} & 8.288 & 851.2150 & -3.1 & 81.3 & 4013 & $\begin{array}{l}715 \text { (136; RDA(Afz)), } 699 \text { (152; RDA), } 697(136,18 ; \mathrm{RDA} \\
\left.(\mathrm{Afz}), \mathrm{H}_{2} \mathrm{O}\right), 579(272 ; \mathrm{QM} \uparrow), 561(290 ; \mathrm{QM} \downarrow), 543(290,18 ; \\
\left.\mathrm{QM} \downarrow, \mathrm{H}_{2} \mathrm{O}\right), 435\left(272,126,18 ; \mathrm{QM} \uparrow, \mathrm{HRF}, \mathrm{H}_{2} \mathrm{O}\right), 427(272,152 ; \\
\mathrm{QM} \uparrow, \mathrm{RDA}), 409(290,152 ; \mathrm{QM} \downarrow, \mathrm{RDA}), 391(272,152,18 ; \\
\left.\mathrm{QM} \uparrow, \mathrm{RDA}, \mathrm{H}_{2} \mathrm{O}\right), 291(560 ; \mathrm{QM} \uparrow), 289(272,290 ; \mathrm{QM} \uparrow, \\
\mathrm{QM} \downarrow), 273(578 ; \mathrm{QM} \downarrow)\end{array}$ & $\begin{array}{l}\text { (epi)afz-(epi)cat- } \\
\text { (epi)cat }\end{array}$ \\
\hline \multirow{4}{*}{ Crataegus EA } & B-type & {$\left[\mathrm{C}_{45} \mathrm{H}_{39} \mathrm{O}_{18}\right]^{+}$} & 9.519 & 867.2143 & 1.2 & 9.8 & 35244 & $\begin{array}{l}\left.741 \text { (126; HRF), } 715 \text { (152; RDA), } 697 \text { (152, 18; RDA, } \mathrm{H}_{2} \mathrm{O}\right), 579 \\
(288 ; \mathrm{QM} \uparrow), 577 \text { (290; QM } \downarrow), 451(126,290 ; \mathrm{HRF}, \mathrm{QM} \downarrow), 427 \\
(288,152 ; \mathrm{QM} \uparrow, \mathrm{RDA}), 425(290,152 ; \mathrm{QM} \downarrow, \mathrm{RDA}), 409(288, \\
\left.\text { 152, 18; QM } \uparrow, \mathrm{RDA}, \mathrm{H}_{2} \mathrm{O}\right), 407(290,152,18 ; \mathrm{QM} \downarrow, \mathrm{RDA}, \\
\left.\mathrm{H}_{2} \mathrm{O}\right), 291(576 ; \mathrm{QM} \uparrow), 289(578 ; \mathrm{QM} \downarrow)\end{array}$ & $\begin{array}{l}\text { (epi)cat-(epi)cat- } \\
\text { (epi)cat }\end{array}$ \\
\hline & A-type & {$\left[\mathrm{C}_{45} \mathrm{H}_{37} \mathrm{O}_{18}\right]^{+}$} & 8.552 & 865.1982 & -0.8 & $-*$ & 7540 & $\begin{array}{l}\left.713 \text { (152; RDA), } 695\left(152,18 ; \mathrm{RDA}, \mathrm{H}_{2} \mathrm{O}\right), 577 \text { (288; QM } \uparrow\right), \\
\left.407 \text { (288, 152, 18; QM } \uparrow, \mathrm{RDA}, \mathrm{H}_{2} \mathrm{O}\right), 289(576 ; \mathrm{QM} \downarrow), 287 \text { (288, } \\
290 ; \mathrm{QM} \uparrow, \text { A-type-QM } \downarrow)\end{array}$ & $\begin{array}{l}\text { (epi)cat-(epi)cat- } \\
\text { A-(epi)cat }\end{array}$ \\
\hline & $\begin{array}{l}\text { B-type with } \\
\text { one } \\
\text { (epi)afzelechin }\end{array}$ & {$\left[\mathrm{C}_{45} \mathrm{H}_{39} \mathrm{O}_{17}\right]^{+}$} & 8.394 & 851.2182 & -0.4 & 41.0 & 6338 & $\begin{array}{l}715(136 ; \mathrm{RDA}(\mathrm{Afz})), 699(152 ; \mathrm{RDA}), 697(136,18 ; \mathrm{RDA} \\
\left.(\mathrm{Afz}), \mathrm{H}_{2} \mathrm{O}\right), 579(272 ; \mathrm{QM} \uparrow), 561(290 ; \mathrm{QM} \downarrow), 543(290,18 ; \\
\left.\mathrm{QM} \downarrow, \mathrm{H}_{2} \mathrm{O}\right), 435\left(272,126,18 ; \mathrm{QM} \uparrow, \mathrm{HRF}, \mathrm{H}_{2} \mathrm{O}\right), 427(272,152 ; \\
\mathrm{QM} \uparrow, \mathrm{RDA}), 409(290,152 ; \mathrm{QM} \downarrow, \mathrm{RDA}), 391(272,152,18 ; \\
\left.\mathrm{QM} \uparrow, \mathrm{RDA}, \mathrm{H}_{2} \mathrm{O}\right), 291(560 ; \mathrm{QM} \uparrow), 289(272,290 ; \mathrm{QM} \uparrow, \\
\mathrm{QM} \downarrow), 273(578 ; \mathrm{QM} \downarrow)\end{array}$ & $\begin{array}{l}\text { (epi)afz-(epi)cat- } \\
\text { (epi)cat }\end{array}$ \\
\hline & $\begin{array}{l}\text { B-type with } \\
\text { two } \\
\text { (epi)afzelechin }\end{array}$ & {$\left[\mathrm{C}_{45} \mathrm{H}_{39} \mathrm{O}_{16}\right]^{+}$} & 7.429 & 835.2248 & 1.6 & 27.1 & 5916 & $\begin{array}{l}\left.699 \text { (136, RDA(Afz)), } 681 \text { (136, 18; RDA (Afz), } \mathrm{H}_{2} \mathrm{O}\right), 665 \text { (152, } \\
\text { 18; RDA, H2O), } 563 \text { (272; QM } \uparrow), 545 \text { (290; QM } \downarrow), 527(290,18 ; \\
\left.\mathrm{QM} \downarrow, \mathrm{H}_{2} \mathrm{O}\right), 427(272,136 ; \mathrm{QM} \uparrow, \mathrm{RDA}(\mathrm{Afz})), 411(272,152 ; \\
\mathrm{QM} \uparrow, \mathrm{RDA}), 409(290,136 ; \mathrm{QM} \downarrow, \mathrm{RDA}(\mathrm{Afz})), 393(290,152 ; \\
\mathrm{QM} \downarrow, \mathrm{RDA}), 291(544, \mathrm{QM} \uparrow), 273(562, \mathrm{QM} \downarrow)\end{array}$ & $\begin{array}{l}\text { (epi)afz-(epi)afz- } \\
\text { (epi)cat }\end{array}$ \\
\hline
\end{tabular}




\begin{tabular}{|c|c|c|c|c|c|c|c|c|c|}
\hline & Cinchonain & {$\left[\mathrm{C}_{54} \mathrm{H}_{45} \mathrm{O}_{21}\right]^{+}$} & 10.043 & 1029.2448 & 0.0 & 49.1 & 5587 & $\begin{array}{l}877 \text { (152; RDA), } 859 \text { (152,18; RDA, H2O), } 739(290 ; \mathrm{QM} \downarrow), 721 \\
\left(290,18 ; \mathrm{QM} \downarrow, \mathrm{H}_{2} \mathrm{O}\right) 629(290,110 ; \mathrm{QM} \downarrow, \mathrm{Catechol-fission),} \\
587(290,152 ; \mathrm{QM} \downarrow, \mathrm{RDA}), 579(450 ; \mathrm{QM} \uparrow), 451(578 ; \mathrm{QM} \downarrow), \\
409(450,152,18 ; \mathrm{QM} \uparrow, \mathrm{RDA}, \mathrm{H} 2 \mathrm{O}), 291(450,288 ; \\
\mathrm{QM} \uparrow, \mathrm{QM} \uparrow), 289(450,290 ; \mathrm{QM} \uparrow, \mathrm{QM} \downarrow)\end{array}$ & $\begin{array}{l}\text { Cinchonain- } \\
\text { (epi)cat-(epi)cat- } \\
\text { (epi)cat }\end{array}$ \\
\hline \multirow{7}{*}{ Wisteria EA } & B-type AAA & {$\left[\mathrm{C}_{45} \mathrm{H}_{39} \mathrm{O}_{15}\right]^{+}$} & 6.456 & 819.2248 & 3.6 & 17.6 & 3778 & $\begin{array}{l}\left.683 \text { (136; RDA), } 665 \text { (136, 18; RDA, } \mathrm{H}_{2} \mathrm{O}\right), 547(272 ; \mathrm{QM} \uparrow), \\
545 \text { (274; QM } \downarrow), 527\left(274,18 ; \mathrm{QM} \downarrow, \mathrm{H}_{2} \mathrm{O}\right), 421(272,126 ; \\
\mathrm{QM} \uparrow, \mathrm{HRF}), 411(272,136 ; \mathrm{QM} \uparrow, \mathrm{RDA}), 409(274,136 ; \mathrm{QM} \downarrow \\
\mathrm{RDA}), 393\left(272,136,18 ; \mathrm{QM} \uparrow, \mathrm{RDA}, \mathrm{H}_{2} \mathrm{O}\right), 391(274,136,18 ; \\
\left.\mathrm{QM} \downarrow, \mathrm{RDA}, \mathrm{H}_{2} \mathrm{O}\right), 273(546 ; \mathrm{QM} \downarrow), 275(544 ; \mathrm{QM} \uparrow)\end{array}$ & $\begin{array}{l}\text { (epi)afz-(epi)afz- } \\
\text { (epi)afz }\end{array}$ \\
\hline & B-type AAC & {$\left[\mathrm{C}_{45} \mathrm{H}_{39} \mathrm{O}_{16}\right]^{+}$} & 7.537 & 835.2200 & -3.2 & 18.6 & 14253 & \begin{tabular}{|l|}
699 (136, RDA(Afz)), 681 (136, 18; RDA (Afz), $\left.\mathrm{H}_{2} \mathrm{O}\right), 665$ (152, \\
$\left.18 ; \mathrm{RDA}, \mathrm{H}_{2} \mathrm{O}\right), 563(272 ; \mathrm{QM} \uparrow), 545$ (290; QM $\left.\downarrow\right), 527(290,18 ;$ \\
$\left.\mathrm{QM} \downarrow, \mathrm{H}_{2} \mathrm{O}\right), 427(272,136 ; \mathrm{QM} \uparrow, \mathrm{RDA}(\mathrm{Afz})), 411(272,152 ;$ \\
$\mathrm{QM} \uparrow, \mathrm{RDA}), 409(290,136 ; \mathrm{QM} \downarrow, \mathrm{RDA}(\mathrm{Afz})), 393(290,152 ;$ \\
$\mathrm{QM} \downarrow, \mathrm{RDA}), 291(544, \mathrm{QM} \uparrow), 273(562, \mathrm{QM} \downarrow)$
\end{tabular} & $\begin{array}{l}\text { (epi)afz-(epi)afz- } \\
\text { (epi)cat }\end{array}$ \\
\hline & B-type ACC & {$\left[\mathrm{C}_{45} \mathrm{H}_{39} \mathrm{O}_{17}\right]^{+}$} & 8.647 & 851.2137 & -4.5 & 30.5 & 13957 & $\begin{array}{l}715(136 ; \mathrm{RDA}(\mathrm{Afz})), 699(152 ; \mathrm{RDA}), 697(136,18 ; \mathrm{RDA} \\
\left.(\mathrm{Afz}), \mathrm{H}_{2} \mathrm{O}\right), 579(272 ; \mathrm{QM} \uparrow), 561(290 ; \mathrm{QM} \downarrow), 543(290,18 ; \\
\left.\mathrm{QM} \downarrow, \mathrm{H}_{2} \mathrm{O}\right), 435\left(272,126,18 ; \mathrm{QM} \uparrow, \mathrm{HRF}, \mathrm{H}_{2} \mathrm{O}\right), 427(272,152 ; \\
\mathrm{QM} \uparrow, \mathrm{RDA}), 409(290,152 ; \mathrm{QM} \downarrow, \mathrm{RDA}), 391(272,152,18 ; \\
\left.\mathrm{QM} \uparrow, \mathrm{RDA}, \mathrm{H}_{2} \mathrm{O}\right), 291(560 ; \mathrm{QM} \uparrow), 289(272,290 ; \mathrm{QM} \uparrow, \\
\mathrm{QM} \downarrow), 273(578 ; \mathrm{QM} \downarrow)\end{array}$ & $\begin{array}{l}\text { (epi)afz-(epi)cat- } \\
\text { (epi)cat }\end{array}$ \\
\hline & B-type CCC & {$\left[\mathrm{C}_{45} \mathrm{H}_{39} \mathrm{O}_{18}\right]^{+}$} & 9.411 & 867.2092 & 3.9 & 63.6 & 8877 & $\begin{array}{l}\left.715 \text { (152; RDA), } 697 \text { (152, 18; RDA, } \mathrm{H}_{2} \mathrm{O}\right), 579(288 ; \mathrm{QM} \uparrow), \\
577(290 ; \mathrm{QM} \downarrow), 451(126,290 ; \mathrm{HRF}, \mathrm{QM} \downarrow), 427(288,152 ; \\
\mathrm{QM} \uparrow, \mathrm{RDA}), 425(290,152 ; \mathrm{QM} \downarrow, \mathrm{RDA}), 409(288,152,18 ; \\
\left.\mathrm{QM} \uparrow, \mathrm{RDA}, \mathrm{H}_{2} \mathrm{O}\right), 407\left(290,152,18 ; \mathrm{QM} \downarrow, \mathrm{RDA}, \mathrm{H}_{2} \mathrm{O}\right), 291 \\
(576 ; \mathrm{QM} \uparrow), 289(578 ; \mathrm{QM} \downarrow)\end{array}$ & $\begin{array}{l}\text { (epi)cat-(epi)cat- } \\
\text { (epi)cat }\end{array}$ \\
\hline & A-type ACC & {$\left[\mathrm{C}_{45} \mathrm{H}_{37} \mathrm{O}_{17}\right]^{+}$} & 8.029 & 849.1989 & 3.7 & -* & 3694 & \multicolumn{2}{|c|}{ Quality of $\mathrm{MS}^{2}$ spectra is not sufficient for examining distinct PAC structure } \\
\hline & A-type CCC & {$\left[\mathrm{C}_{45} \mathrm{H}_{37} \mathrm{O}_{18}\right]^{+}$} & 9.014 & 865.1931 & 4.3 & $-*$ & 4675 & \multicolumn{2}{|c|}{ Quality of $\mathrm{MS}^{2}$ spectra is not sufficient for examining distinct PAC structure } \\
\hline & A-type CCG & {$\left[\mathrm{C}_{45} \mathrm{H}_{37} \mathrm{O}_{19}\right]^{+}$} & 10.112 & 881.1885 & -3.8 & $-*$ & 3925 & \multicolumn{2}{|c|}{ Quality of $\mathrm{MS}^{2}$ spectra is not sufficient for examining distinct PAC structure } \\
\hline \multirow{3}{*}{ Rumex EA } & $\begin{array}{l}\text { B-type DP } 2 \\
\text { digallate }\end{array}$ & {$\left[\mathrm{C}_{44} \mathrm{H}_{35} \mathrm{O}_{20}\right]^{+}$} & 9.983 & 883.1732 & 1.6 & 51.2 & 24973 & \begin{tabular}{|l|}
757 (126; HRF), 713 (170; GF), 591 (292, 543 (170, 170; \\
GF,GF), 579 (170,134; GF, RDA(after GF)), 441 (170, 272; GF, \\
QM $\uparrow), 271$ (170, 170, 272; GF, GF, QM)
\end{tabular} & $\begin{array}{l}\text { (epi)cat-gallate- } \\
\text { (epi)cat-gallate }\end{array}$ \\
\hline & B-type ACC & {$\left[\mathrm{C}_{45} \mathrm{H}_{39} \mathrm{O}_{17}\right]^{+}$} & 8.572 & 851.2145 & -4.0 & -4.7 & 44.7 & \begin{tabular}{|l|}
715 (136; RDA(Afz)), 579 (272; QM $\uparrow), 561(290 ; \mathrm{QM} \downarrow), 543$ \\
$\left(290,18 ; \mathrm{QM} \downarrow, \mathrm{H}_{2} \mathrm{O}\right), 409\left(288,152,18 ; \mathrm{QM} \uparrow, \mathrm{RDA}, \mathrm{H}_{2} \mathrm{O}\right), 289$ \\
$(578 ; \mathrm{QM} \downarrow)$
\end{tabular} & $\begin{array}{l}\text { (epi)afz-(epi)cat- } \\
\text { (epi)cat }\end{array}$ \\
\hline & B-type CCC & {$\left[\mathrm{C}_{45} \mathrm{H}_{39} \mathrm{O}_{18}\right]^{+}$} & 9.865 & 867.2103 & 2.8 & 19.1 & 6969 & \begin{tabular}{|l|}
741 (126; HRF), 715 (152; RDA), 697 (152, 18; RDA, $\left.\mathrm{H}_{2} \mathrm{O}\right), 579$ \\
$(288 ; \mathrm{QM} \uparrow), 577(290 ; \mathrm{QM} \downarrow), 451(126,290 ; \mathrm{HRF}, \mathrm{QM} \downarrow), 427$ \\
$(288,152 ; \mathrm{QM} \uparrow, \mathrm{RDA}), 425(290,152 ; \mathrm{QM} \downarrow, \mathrm{RDA}), 409(288$, \\
152, 18; QM $\left.\uparrow, \mathrm{RDA}, \mathrm{H}_{2} \mathrm{O}\right), 407(290,152,18 ; \mathrm{QM} \downarrow, \mathrm{RDA}$, \\
$\left.\mathrm{H}_{2} \mathrm{O}\right), 291(576 ; \mathrm{QM} \uparrow), 289(578 ; \mathrm{QM} \downarrow)$
\end{tabular} & \begin{tabular}{|l} 
(epi)cat-(epi)cat- \\
(epi)cat
\end{tabular} \\
\hline
\end{tabular}




\begin{tabular}{|c|c|c|c|c|c|c|c|c|}
\hline $\begin{array}{l}\text { B-type ACC } \\
\text { monogallate }\end{array}$ & {$\left[\mathrm{C}_{52} \mathrm{H}_{43} \mathrm{O}_{21}\right]^{+}$} & 10.356 & 1003.2283 & 0.8 & 0.8 & 78.5 & $\begin{array}{l}851 \text { (152; RDA), } 833 \text { (170, GF), } 713 \text { (290; QM } \downarrow), 579 \text { (403, } \\
\text { QM } \uparrow \text { (Afz-gallate), } 543 \text { (290, 170; QM } \downarrow \text {, GF), } 425 \text { (290, 288; } \\
\text { QM } \downarrow, \text { QM } \downarrow)\end{array}$ & $\begin{array}{l}\text { (epi)afz-gallate- } \\
\text { (epi)cat-(epi)cat }\end{array}$ \\
\hline $\begin{array}{l}\text { B-type } \\
\text { monogallate }\end{array}$ & {$\left[\mathrm{C}_{52} \mathrm{H}_{43} \mathrm{O}_{22}\right]^{+}$} & 10.916 & 1019.2236 & 0.4 & 35.7 & 5698 & $\begin{array}{l}867(152 ; \mathrm{RDA}), 849(170 ; \mathrm{GF}), 729(290 ; \mathrm{QM} \downarrow), 559(290,170 ; \\
\mathrm{QM} \downarrow, \mathrm{GF}), 441(290,288, \mathrm{QM} \downarrow, \mathrm{QM} \downarrow), 289(290,441 ; \mathrm{QM} \uparrow, \\
\mathrm{QM} \downarrow), 271(170,578 ; \mathrm{GF}, \mathrm{QM} \downarrow)\end{array}$ & $\begin{array}{l}\text { (epi)cat -gallate - } \\
\text { (epi)cat -(epi)cat }\end{array}$ \\
\hline \begin{tabular}{|l|} 
B-type \\
digallate
\end{tabular} & {$\left[\mathrm{C}_{59} \mathrm{H}_{47} \mathrm{O}_{26}\right]^{+}$} & 12.232 & 1171.2387 & 3.7 & 40.0 & 20136 & $\begin{array}{l}1001 \text { (170; GF), } 883(288 ; \mathrm{QM} \uparrow), 729(442 ; \mathrm{QM} \downarrow), 559(442, \\
170 ; \mathrm{QM} \downarrow, \mathrm{GF}), 441(288,442 ; \mathrm{QM} \uparrow, \mathrm{QM} \downarrow), 289(882 ; \mathrm{QM} \downarrow), \\
271(442,288,170 ; \mathrm{QM} \downarrow, \mathrm{QM} \uparrow, \mathrm{GF})\end{array}$ & $\begin{array}{l}\text { (epi)cat -(epi)cat- } \\
\text { gallate -(epi)cat- } \\
\text { gallate }\end{array}$ \\
\hline A-type & {$\left[\mathrm{C}_{45} \mathrm{H}_{37} \mathrm{O}_{18}\right]^{+}$} & 8.713 & 865.1983 & -0.8 & 67.0 & 45536 & $\begin{array}{l}713 \text { (152; RDA), } 695 \text { (152, 18; RDA, H2O), } 575 \text { (290; QM } \downarrow), \\
289(576 ; \mathrm{QM} \downarrow), 287(288,290 ; \mathrm{QM} \uparrow, \mathrm{A} \text {-type-QM } \downarrow)\end{array}$ & $\begin{array}{l}\text { (epi)cat-A- } \\
\text { (epi)cat-(epi)cat }\end{array}$ \\
\hline \begin{tabular}{|l|} 
A-type \\
monogallate
\end{tabular} & {$\left[\mathrm{C}_{52} \mathrm{H}_{41} \mathrm{O}_{22}\right]^{+}$} & 10.198 & 1017.2098 & 1.4 & $-*$ & 5391 & $\begin{array}{l}\left.\left.865 \text { (152; RDA), } 847 \text { (152, 18; RDA, } \mathrm{H}_{2} \mathrm{O}\right), 727 \text { (290; QM } \downarrow\right), \\
439 \text { (578; QM } \downarrow \text { (A-typ)), } 287 \text { ((578, 152; QM } \downarrow \text { (A-typ), GF } \\
\text { mild) }\end{array}$ & $\begin{array}{l}\text { (epi)cat-gallate-A- } \\
\text { (epi)cat-(epi)cat }\end{array}$ \\
\hline \begin{tabular}{|l} 
A-type \\
digallate
\end{tabular} & {$\left[\mathrm{C}_{59} \mathrm{H}_{45} \mathrm{O}_{26}\right]^{+}$} & 11.781 & 1169.2284 & 9.1 & $-*$ & 2135 & \multicolumn{2}{|c|}{ Quality of MS ${ }^{2}$ spectra is not sufficient for examining distinct PAC structure } \\
\hline B-type DP4 & {$\left[\mathrm{C}_{60} \mathrm{H}_{51} \mathrm{O}_{24}\right]^{+}$} & 12.080 & 1155.2753 & -1.2 & 24.2 & 9035 & \multicolumn{2}{|c|}{$\begin{array}{l}1003(152 ; \mathrm{RDA}), 867(288 ; \mathrm{QM} \uparrow), 579(288,288 ; \mathrm{QM} \uparrow, \mathrm{QM} \uparrow), \\
577(288,290 ; \mathrm{QM} \uparrow, \mathrm{QM} \downarrow), 425(288,290,152 ; \mathrm{QM} \uparrow, \mathrm{QM} \downarrow, \\
\text { RDA), } 289(866 ; \mathrm{QM} \downarrow), 291(864 ; \mathrm{QM} \uparrow)\end{array}$} \\
\hline
\end{tabular}

